\title{
The JUNO experiment and its electronics readout system
}

\author{
Barbara Clerbaux* \\ IIHE(ULB-VUB), Université libre de Bruxelles, ULB, Brussels, Belgium \\ E-mail: barbara.clerbauxaulb.ac.be

\section{Yifan Yang} \\ IIHE(ULB-VUB), Université libre de Bruxelles, ULB, Brussels, Belgium \\ E-mail: yifan.yang@ulb.ac.be
}

On behalf of the JUNO Collaboration

The Jiangmen Underground Neutrino Observatory (JUNO) is a neutrino medium baseline experiment in construction in China, with the main goal to determine the neutrino mass hierarchy and to measure several neutrino mass and mixing parameters with a precision at the sub-percent level. The overall picture of the main parts of the JUNO detector, as well as its electronics readout system are presented in this contribution.

European Physical Society Conference on High Energy Physics - EPS-HEP2019 -

10-17 July, 2019

Ghent, Belgium

${ }^{*}$ Speaker. 


\section{Introduction}

The Jiangmen Underground Neutrino observatory (JUNO) [1] experiment is a large liquid scintillator detector aiming at measuring antineutrinos issued from nuclear reactors at a distance of about $53 \mathrm{~km}$, as shown in Fig. 1 (Left). The precise measurement of the antineutrino energy spectrum will allow determining the neutrino mass hierarchy $(\mathrm{NMH})$ and reducing the uncertainty below $1 \%$ on solar oscillation parameters. The required energy resolution to discriminate between the normal and inverted $\mathrm{NMH}$ at a 3-4 $\sigma$ for about 6 years of data taking is 3\% at an energy of $1 \mathrm{MeV}$. The expected energy spectrum to be measured by JUNO is presented in Fig. 1 (Right). The JUNO detector is also capable of observing neutrinos/antineutrinos from terrestrial and extra-terrestrial sources, including geoneutrinos, atmospheric neutrinos, solar neutrinos, supernova neutrinos, and diffuse supernova neutrino background. In addition, sterile neutrinos with $10^{-5}<\Delta m_{41}^{2}<10^{-2}$ $\mathrm{eV}^{2}$ and a sufficiently large mixing angle $\theta_{41}$ could be identified through a precise measurement of the antineutrino energy spectrum. As a result of JUNO's large size, excellent energy resolution and vertex reconstruction capability, interesting new data on these topics will be collected. More details on the science potential of JUNO can be found in Ref. [1].
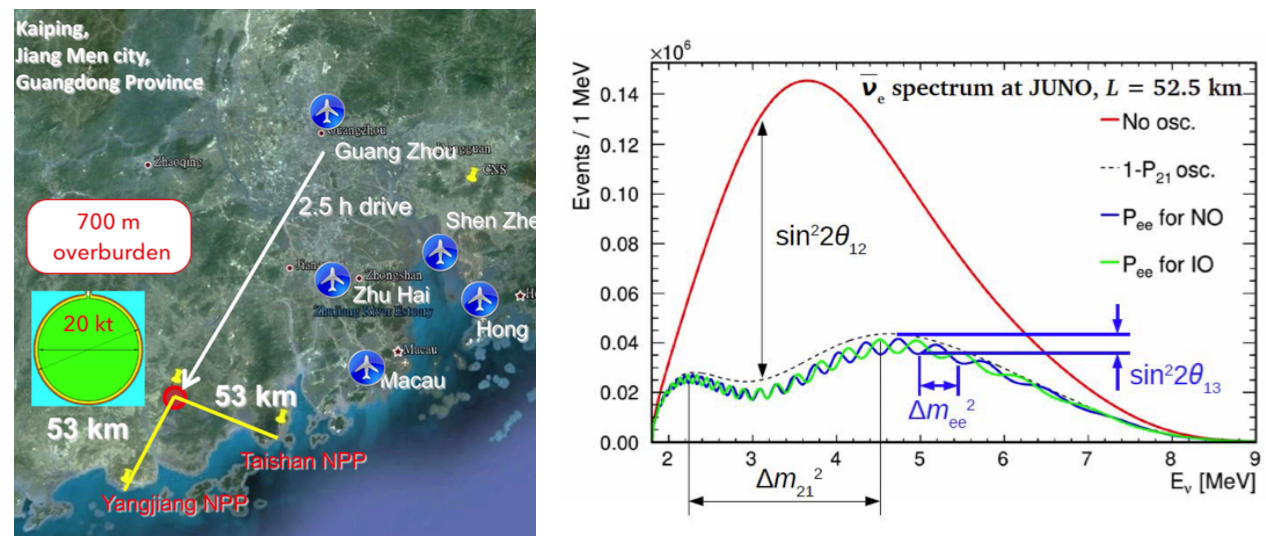

Figure 1: Left : Map of the Kaiping region (Guangdong province, China) where the JUNO detector will be located, at about $53 \mathrm{~km}$ from two nuclear power plants; Right : Expected energy spectrum to be measured by JUNO in the case of no oscillation (in red), normal (black) and inverted (green) neutrino mass hierarchy.

The option to add a smaller detector (2.6 tons of Gd-loaded liquid scintillator, read with SiPMs), called TAO (Taishan Antineutrino Observatory) located at $40 \mathrm{~m}$ distance from the Taishan reactor is being considered and studied. The aim of TAO is to measure the antineutrino spectrum at the percent level, in order to provide a model-independent reference spectrum for JUNO and a benchmark for investigation of the nuclear database.

The JUNO collaboration includes about 600 scientists, from 77 institutions, in 17 countries. The international JUNO collaboration was established in 2014. The civil construction and the PMT production started in 2015 and 2016, respectively, and are expected to end in 2020. In years 202021 , the detector assembly and installation will take place. The liquid scintillator filling is foreseen to happen in 2021. The start of the data taking is expected at the end of 2021/beginning of 2022. 


\section{The JUNO detector}

The requirements on energy resolution (3\% at $1 \mathrm{MeV}$ ) and energy scale (better than $1 \%$ ) put strong constraints on the detector size and on its component quality. A large volume of liquid scintillator is needed, with a high attenuation length. A high photocathode coverage implies an important number of the large PMT to be deployed. To remove background as much as possible, vertex reconstruction capabilities need to be on place. The detector performance goals are listed in Fig. 2 (last column), and are compared to the ones from the Kamland experiment (second column).

\begin{tabular}{|c|c|c|}
\hline Energy Resolution & $6 \% @ 1 \mathrm{MeV}$ & $3 \%$ @ $1 \mathrm{MeV}$ \\
\hline LS mass & $\sim 1 \mathrm{kt}$ & $\mathbf{2 0 ~ \mathbf { ~ t }}$ \\
\hline LS Attenuation/Diameter & $15 \mathrm{~m} / 16 \mathrm{~m}$ & $\mathbf{2 0 \mathrm { m } / 3 5 \mathrm { m }}$ \\
\hline Photocathode Coverage & $32 \%$ & $\mathbf{7 5 \%}$ \\
\hline QE X CE & $25 \% \times 60 \%$ & $\mathbf{4 0 \%} \times \mathbf{6 0} \%$ \\
\hline Photon collection & 250 p.e./MeV & 1200 p.e./MeV \\
\hline
\end{tabular}

Figure 2: The expected detector performance of JUNO (last column) compared to the one of the Kamland experiment (second column).

The JUNO detector will be located at $700 \mathrm{~m}$ underground, and consists of a central detector, an active veto system and a calibration system. The central detector is composed by a $35 \mathrm{~m}$ diameter transparent acrylic sphere, filled by 20 ktons of liquid scintillator (LS), being the antineutrino target. The LS consists of Linear Alkyl Benzene (LAB) as solvent, $3 \mathrm{~g} / \mathrm{L}$ 2,5-diphenyloxazole (PPO) as the fluor and $15 \mathrm{mg} / \mathrm{L} \mathrm{p}$-bis-(o-methylstyryl)-benzene (bis-MSB) as the wavelength shifter. The stainless steel support structure holds the inner vessel and the photomultiplier tubes (PMTs). Dynode and micro-channel plate PMTs are used to detect the scintillation photons from inverse beta decay (IBD) events in the LS. The acrylic sphere is instrumented by more than 1800020 -inch PMT, and about 25000 3-inch small PMTs located in between the large PMTs. The 20-inch PMT's detection efficiency (the product of the quantum efficiency times the collection efficiency) is required to be $27 \%$ on average and $24 \%$ on minimum. The 3 -inch PMTs serve as an additional standalone calorimetry which will not face saturation effect and will provide handle to control the non-linearity effects.

Two vetoes are foreseen to reduce the different backgrounds. A 20 ktons ultrapure water Cerenkov pool around the central detector instrumented by about 200020 -inch PMTs will tag events coming from outside the neutrino target. It will also act as a passive shielding for neutrons and gammas. In addition a muon tracker will be installed on top of the detector (top muon veto) in order to tag cosmic muons and validate the muon track reconstruction. The top muon veto will use the target tracker detector previously used in the OPERA experiment at Gran Sasso. The JUNO detector is detailed in Ref. [2]. A schematic view of the detector is presented in Fig. 3. The fontend and readout electronic for the large PMT system are an important component of the experiment and their performance is crucial for the success of the various JUNO measurements. 
A sophisticated calibration complex is designed for multiple source deployment, the entire energy coverage of reactor neutrinos and for central detector full-volume coverage. Four different calibration systems will be used: an automatic calibration unit (vertical scan), a cable loop system (complete vertical plane scan), a guide tube control system (vertical plane scan from outside of the acrylic sphere) and finally a remotely operated vehicles which will transport a source at any place in the liquid scintillator.
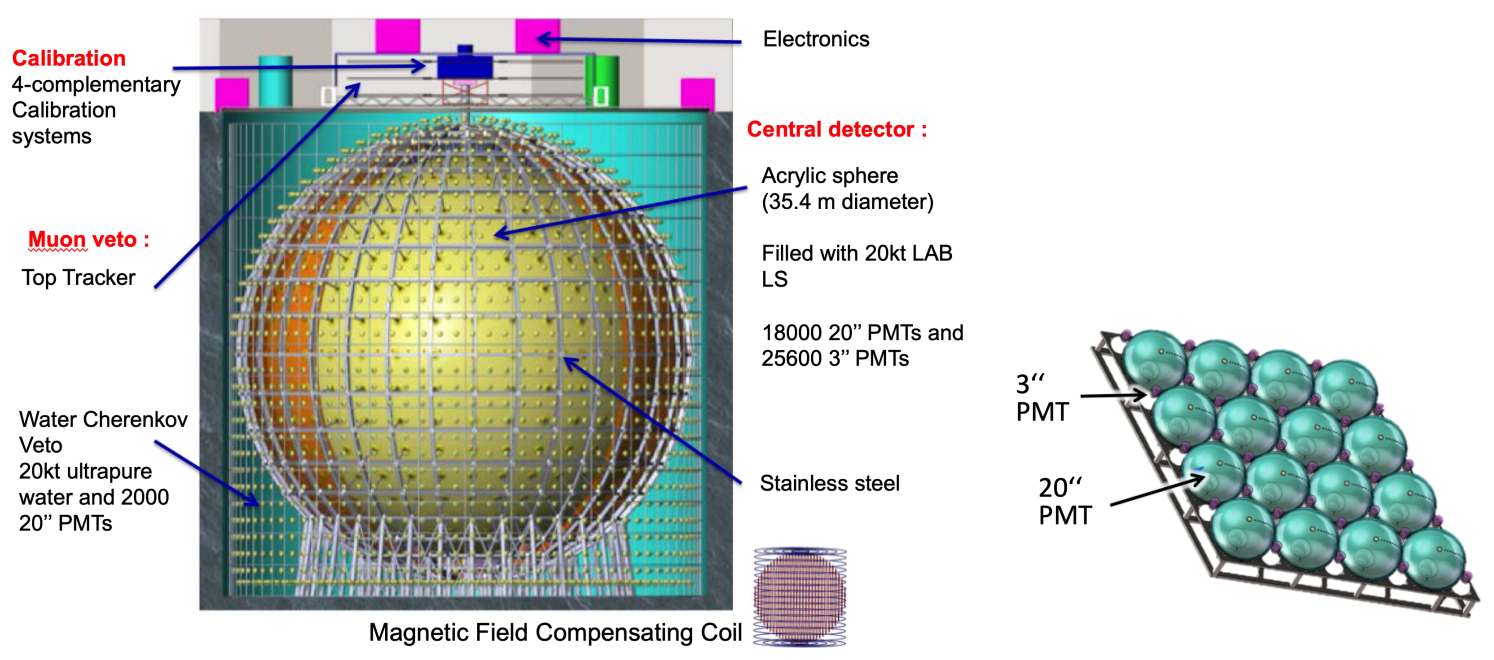

Figure 3: Left : the JUNO detector with the central acrylic sphere, the two veto systems and the calibration part; Right : illustration of the large (20-inch) and small (3-inch) PMT arrangement.

\section{3. the JUNO electronics readout}

The physics requirement on the excellent energy resolution especially at low energy for the NMH measurement implies various specifications on the electronics : excellent photon's arrival time measurement for good vertexing, a large dynamic range (for atm-, geo-, and supernova neutrinos, a negligible deadtime (for supernova events lasting up to few seconds). The following specifications have been defined : provide full waveform digitization with high speed (1 Gsam$\mathrm{ple} / \mathrm{s}$ ), high resolution (nominal resolution of 14 bits) on the full dynamic range: from 1 to $1000 \mathrm{pe}$. The main risk concerns the reliability of the under water electronics that will be not accessible after installation. The reliability requirement to have less than than $1 \%$ PMT and underwater electronics failure over 6 years is studied using complementary methods : direct calculation from the various components, laboratory reliability and ageing tests.

The JUNO electronics system is divided in two main parts: the front-end electronics system performing analog signal processing (the underwater electronics), and after 100 meter Ethernet cables, the backend electronics system, sitting outside water, consisting of the DAQ and the trigger. A schematic view of the electronics readout system of JUNO is given in Fig. 4.

The PMT current signal is conditioned, duplicated in two streams and converted to voltage (low-gain and high-gain TIAs) as follows : for the low gain (8:1) in the case of $0-1000$ pe, and for the high gain (1:1) in the case of 1-128 pe. Each stream is digitized with a 14-bit 1 Gsample/s 


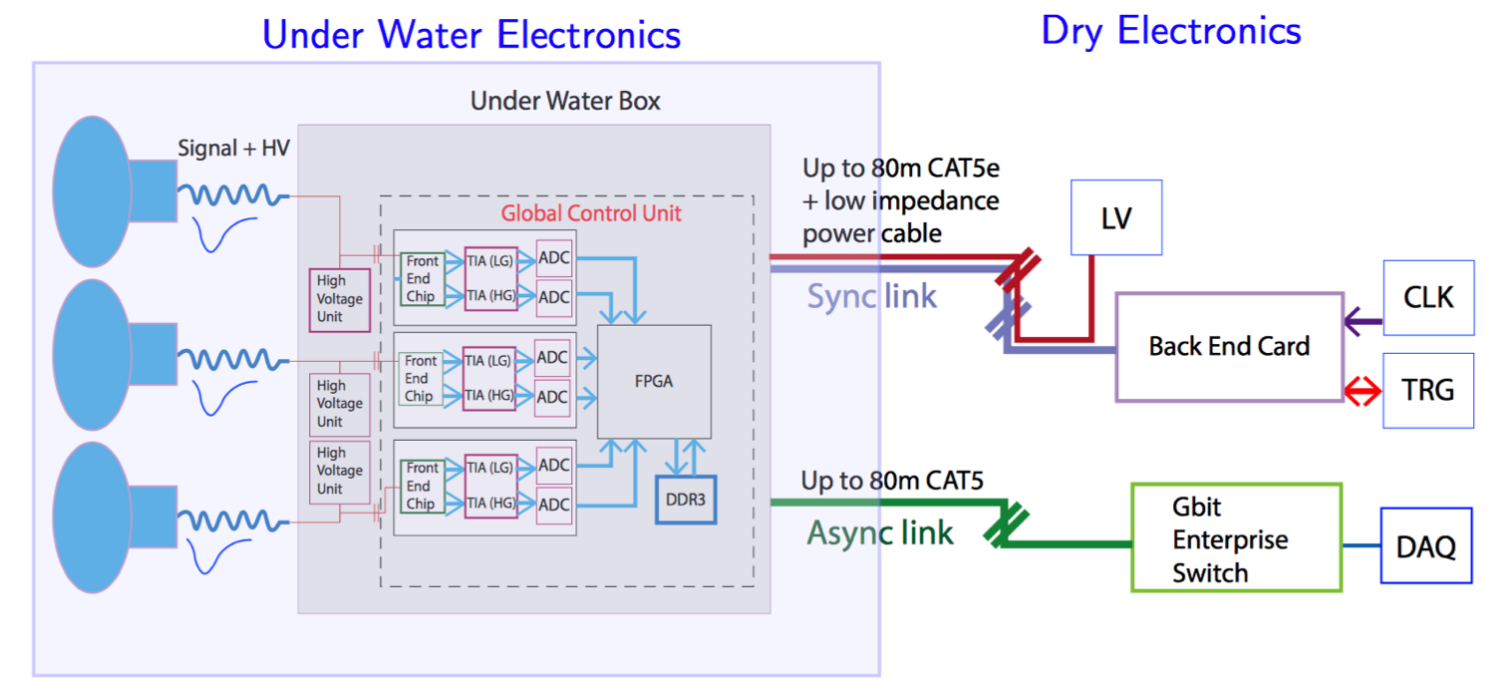

Figure 4: Schematic view of the electronics readout system of JUNO : the underwater part on the left side and the above water part on the right side.

custom designed ASIC, developed by Tsinghua University. Digital signal is then processed in the FPGA (Xilinx kintex7), the reconstructed (timestamp, charge) and the digitized waveform is then stored locally (2 GB DDR RAM). All the Global Control Units (GCUs), about 7000, are synchronised in a $<16$ ns window. See Refs. [3, 4] for more details on the GCUs and on the clock system and timing synchronisation. A picture of the GCU board is presented in Fig. 5.

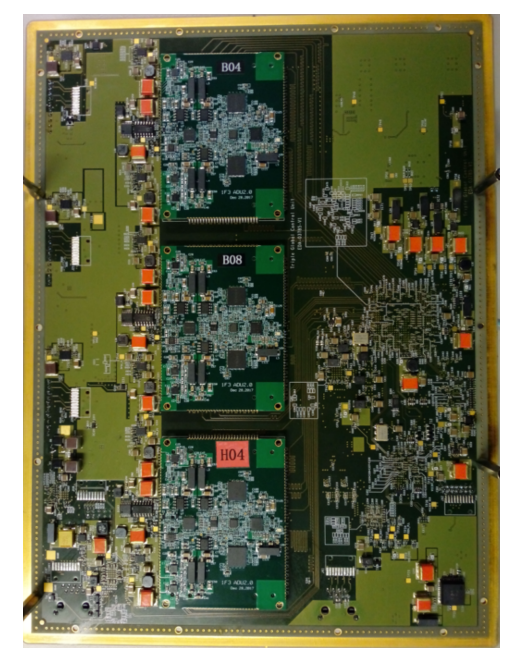

Figure 5: Picture of the Global Control Unit (GCU) with 3 ADU mezzanine cards installed in the middle, each ADU contains 2 ADC for the high gain and low gain PMT signal separately.

In the "Global-Trigger" running mode, a local trigger signal is sent to the Global Trigger and, if validated, data are transferred to DAQ through Ethernet. In the "Auto-Trigger" mode, fixed window waveforms (300 ns) are sent to the DAQ every time a local trigger is issued. 
The digital signal and trigger informations are forwarded to the dry electronics by means of $100 \mathrm{~m}$ CAT5 Ethernet cables. From each under water box, 3 cables are connected to the dry electronics: (1) one CAT5 UTP cable, the variable latency ethernet link (asynchronous link) for data readout and slow control (using the IPBUS protocol and a nominal link speed of 1Gbps); (2) one CAT5 FTP cable, the fixed latency link (synchronous link) to the backend cards (BEC) for the trigger and the clock (using the Timing Trigger and Control (TTC) protocol and a nominal link speed of $125 \mathrm{Mbps}$ ); (3) a low impedance power cable.

For the back-end electronics part, the BEC are used as concentrators to collect and compensate the incoming trigger request signals and an FPGA mezzanine card handles all trigger request signals. Each BEC receives 48 Ethernet cables from the 48 underwater boxes and distributes the clock signal to the GCU's. The signals from the various BECs are sent to 21 RMU (Reorganise\&Multiplex Unit) cards, and their sum is forwarded to the CTU (Central Trigger Unit).
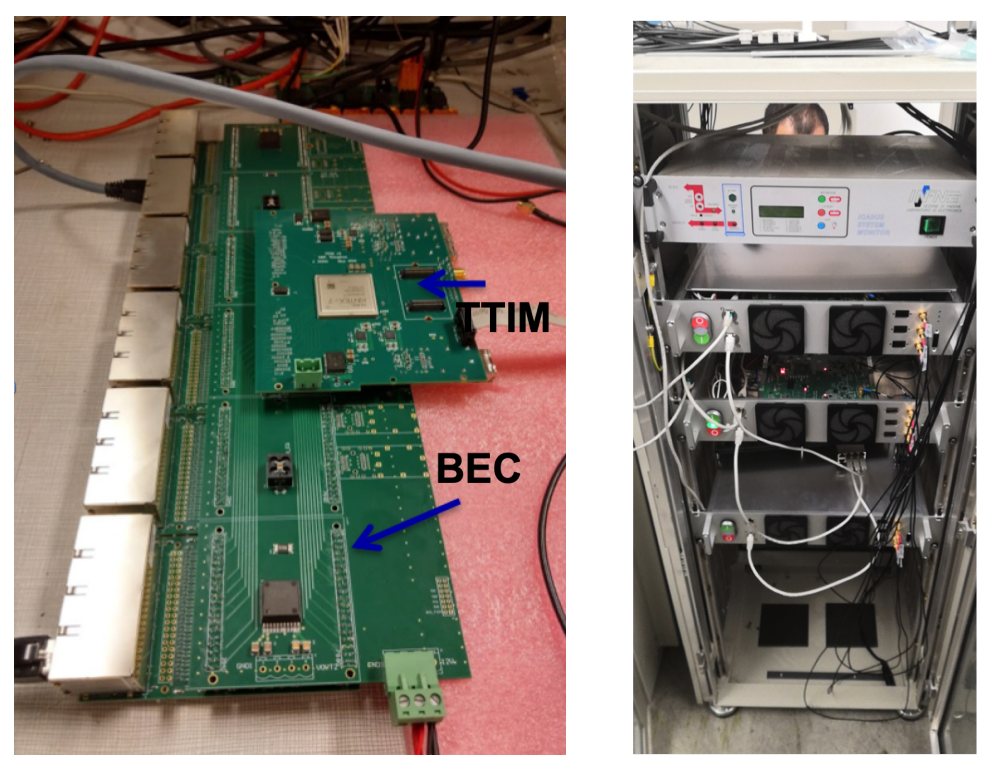

Figure 6: Left : photography of the backend card (BEC version 4, in Brussels, Belgium) composed by the baseboard and the 6 mezzanine cards. On each mezzanine card the 8 connectors are visible on the left side of the picture. The TTIM (Trigger/Timing interface mezzanine) is also visible on top of the baseboard; Right : the setup of the integration test, performed in Padova, Italy, where 3 GCUs are connected to 1 BEC and TTIM.

The readout system of the about 25000 small (3-inch) PMT system is similar to the one of the large (20-inch) PMTs, in particular for the outside water part. Signals from the 128 small PMTs are collected to an underwater box, which includes all the frontend electronics : the high voltage power supplies and splitting signals, the readout and digitization of the signal, which is performed by a electronics board holding 8 CATIROC ASICs, controlled by an FPGA. The CATIROC ASIC allows working on the single photo-electron (p.e.). It provides a charge measurement over a dynamic range from 1 to several hundreds of photoelectrons. The 200 underwater boxes from the small PMTs are connected to the outside water system in the same way as for the large PMT, with a total of 5 BEC needed. 
A schematic view of the global trigger scheme is given in Fig. 7. It is composed of 21 RMU, each of them receiving the signals from 7 BECs. The signals from the 21 RMUs are sent to the 180 vertex fit logic (VFL) units, which are implemented inside the central trigger unit.

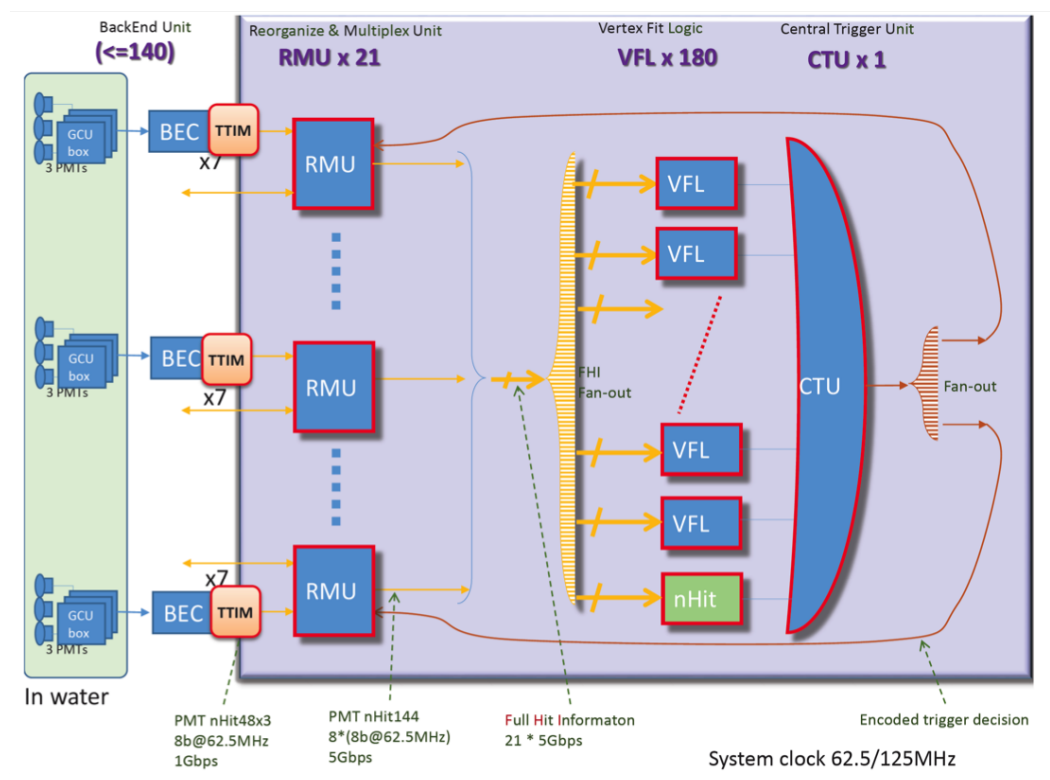

Figure 7: Schematic view of the trigger system of JUNO. It is composed of the RMU (reorganise \& multiplex unit), the vertex fit logic (VFL) and the central trigger unite (CTU).

\section{Conclusions}

JUNO is an unprecedented liquid scintillator detector by its size and by its resolution. The goal is to reach an energy resolution of $3 \%$ at $1 \mathrm{MeV}$. This requirement put strong constraints on the detector subsystems and on the electronics readout chain of the experiment. The design of the electronics is almost completed, all the different components have been validated and a full integration test is being performed. The production of the individual parts will follow.

\section{References}

[1] F. An et al., "Neutrino Physics with JUNO", J. Phys. G43 (2016) 030401, arXiv:1507.05613

[2] T. Adam et al., JUNO Collaboration, "JUNO Conceptual Design Report", arXiv:1508.07166, Instrumentation and Detectors, 328 pages

[3] D. Pedretti et al., "The Global Control Unit for the JUNO front-end electronics", in Liu ZA. (eds) Proceedings of International Conference on Technology and Instrumentation in Particle Physics 2017. TIPP 2017. Springer Proceedings in Physics, vol 212. Springer, Singapore

[4] D. Pedretti et al., "Nanoseconds Timing System Based on IEEE 1588 FPGA Implementation", Published in: IEEE Transactions on Nuclear Science, Volume 66, Issue 7, July 2019, Pages: 1151 1158, Date of Publication: 19 March 2019. ArXiv:1806.04586, DOI: 10.1109/TNS.2019.2906045 